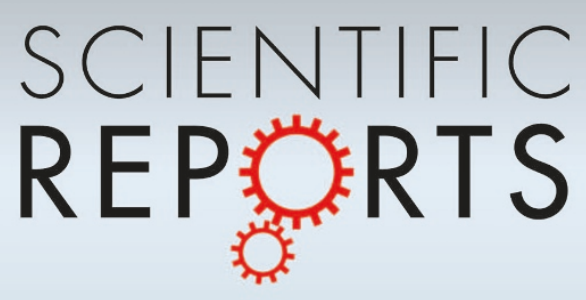

\title{
OPEN \\ Optical conductivity of nodal metals
}

SUBJECT AREAS:

ELECTRONIC PROPERTIES

AND MATERIALS

SUPERCONDUCTING PROPERTIES AND MATERIALS

INFRARED SPECTROSCOPY

TOPOLOGICAL INSULATORS

Received

27 March 2013

Accepted

28 October 2013

Published

13 December 2013

Correspondence and requests for materials should be addressed to

C.C.H. (homes@bnl. gov) or J.J.T. (jłu@sci. cony.cuny.edu)

\author{
C. C. Homes' , J. J. Tu², J. Li², G. D. Gu' \& A. Akrap ${ }^{3}$
}

${ }^{1}$ Condensed Matter Physics and Materials Science Department, Brookhaven National Laboratory, Upton, New York 11973 , USA, ${ }^{2}$ Department of Physics, The City College of New York, New York, New York 10031, USA, 3École de Physique, Université de Genève, $\mathrm{CH}-1211$ Genève 4, Switzerland.

Fermi liquid theory is remarkably successful in describing the transport and optical properties of metals; at frequencies higher than the scattering rate, the optical conductivity adopts the well-known power law behavior $\sigma_{1}(\omega) \propto \omega^{-2}$. We have observed an unusual non-Fermi liquid response $\sigma_{1}(\omega) \propto \omega^{-1 \pm 0.2}$ in the ground states of several cuprate and iron-based materials which undergo electronic or magnetic phase transitions resulting in dramatically reduced or nodal Fermi surfaces. The identification of an inverse (or fractional) power-law behavior in the residual optical conductivity now permits the removal of this contribution, revealing the direct transitions across the gap and allowing the nature of the electron-boson coupling to be probed. The non-Fermi liquid behavior in these systems may be the result of a common Fermi surface topology of Dirac cone-like features in the electronic dispersion.

$\mathrm{n}$ a Fermi liquid, the complex conductivity $\tilde{\sigma}=\sigma_{1}+i \sigma_{2}$ can be expressed through the generalized Drude model, $\tilde{\sigma}(\omega)=\left(\omega_{p}^{2} / 60\right) /\{1 / \tau(\omega)-i \omega[1+\lambda(\omega)]\}$ (in units of $\Omega^{-1} \mathrm{~cm}^{-1}$ ), where $\omega_{p}^{2}=4 \pi n e^{2} / m_{b}, 1 / \tau(\omega)$ and $1+\lambda(\omega)=m^{*}(\omega) / m_{b}$ are the plasma frequency, frequency-dependent scattering rate and mass enhancement, respectively, where $n$ is a carrier concentration and $m_{b}$ is the band mass. At low-temperature the scattering rate will vary quadratically with frequency and temperature, $1 / \tau(\omega, T)=1 / \tau_{0}+A\left[(\hbar \omega)^{2}+\left(2 \pi k_{\mathrm{B}} T\right)^{2}\right]$, where $A$ is a constant that varies with the material ${ }^{1,2}$. In the frequency domain, for $\omega \tau \ll 1$ the conductivity varies slowly, but for $\omega \tau \gg 1$ the conductivity adopts a power-law behavior, $\sigma_{1} \propto \omega^{-2}$; however, deviations from this behavior may be observed in strongly-correlated electronic systems $\mathrm{s}^{3-7}$.

\section{Results}

The temperature dependence of the optical conductivity of optimally-doped $\mathrm{Bi}_{2} \mathrm{Sr}_{2} \mathrm{CaCu}_{2} \mathrm{O}_{8+\delta}$, one of the most thoroughly studied cuprate high-temperature superconductors ${ }^{8}$, is shown versus wave number (photon energy) in a log-log plot in Fig. 1a for light polarized along the crystallographic $a$ axis ${ }^{9}$. Just above $T_{c}$ it may be argued that the optical properties are consistent with those of a Fermi liquid (see Supplementary Information and Fig. S1 online for a discussion of different models for the optical conductivity and the frequency-dependent scattering rate); this statement is in keeping with the proposed phase diagram for the high-temperature superconductors ${ }^{10}$. Below $T_{c}$ there is a rapid reduction of the low-frequency conductivity or spectral weight, which is defined as the area under the conductivity curve; this 'missing spectral weight' is the optical signature for the formation of a superconducting condensate ${ }^{8}$. However, even down to the lowest measured temperature there is still a significant amount of low-frequency residual conductivity ${ }^{11}$. This is because, unlike a conventional $s$-wave superconductor in which the entire Fermi surface is completely gapped below $T_{c}$, the cuprate materials have a momentumdependent $d$-wave gap that contains nodes ${ }^{12,13}, \Delta(\mathbf{k})=\Delta_{0}\left[\cos \left(k_{x} a\right)-\cos \left(k_{y} a\right)\right]$, where $\Delta_{0}$ is the gap maximum. The presence of nodes allows pair-breaking out of the superconducting state resulting in unpaired nodal quasiparticles ${ }^{14}$. For low photon energies $\left(\hbar \omega \ll 2 \Delta_{0}\right)$ only the nodal structure of the $d$-wave gap is probed and the Fermi surface topology is similar to that of the Dirac cone observed in graphene and other quantum materials ${ }^{15,16}$. The rapid collapse of the quasiparticle scattering rate ${ }^{17}$ below $T_{c}$ indicates that in the far-infrared region $\omega \tau \gg 1$, so $\sigma_{1} \propto \omega^{-2}$ should be clearly revealed. Surprisingly, what is observed instead is that below $T_{c}$ low-frequency residual optical conductivity forms a family of lines with the same non-Fermi liquid fractional power law behavior $\sigma_{1} \propto \omega^{-1.2}$; in metallic systems at low-frequency where $\sigma_{1} \gg \sigma_{2}$, this is approximately equivalent to the scattering rate having a fractional power law behavior $1 / \tau \propto \omega^{1.2}$. Another family of cuprates that has been extensively investigated are the $\mathrm{YBa}_{2} \mathrm{Cu}_{3} \mathrm{O}_{6+y}$ materials. The optical conductivity of optimally-doped $\mathrm{YBa}_{2} \mathrm{Cu}_{3} \mathrm{O}_{6.95}$ is shown in Fig. $1 \mathrm{~b}$ for light polarized along the $a$ axis; this crystallographic axis is transverse to the copper-oxygen chains and should therefore probe the dynamics of only the copper-oxygen planes ${ }^{18}$. Well below $T_{c}$, the observed power law for the residual conductivity $\sigma_{1} \propto \omega^{-1.2}$ is identical to the response observed in optimally-doped $\mathrm{Bi}_{2} \mathrm{Sr}_{2} \mathrm{CaCu}_{2} \mathrm{O}_{8+\delta}$. The underdoped $\mathrm{YBa}_{2} \mathrm{Cu}_{3} \mathrm{O}_{6.60}$ sample is of particular interest due to the 

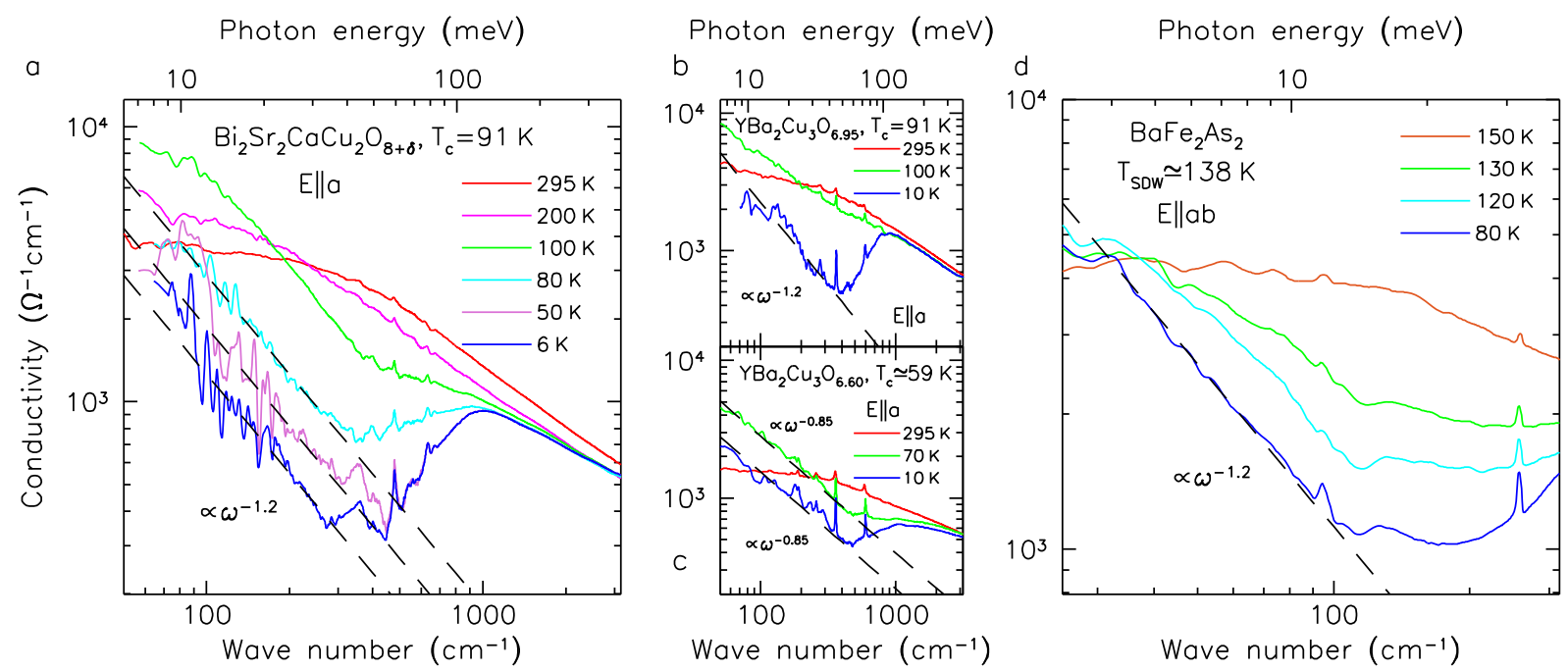

Figure 1 The optical conductivity of some quantum materials. (a), The temperature dependence of the optical conductivity versus wave number (photon energy) for optimally-doped $\mathrm{Bi}_{2} \mathrm{Sr}_{2} \mathrm{CaCu}_{2} \mathrm{O}_{8+\delta}\left(T_{c}=91 \mathrm{~K}\right.$ ) for light polarized along the crystallographic $a$ axis. At low frequency just above $T_{c}$ the material may be cautiously described as a Fermi liquid. For all the temperatures measured below $T_{c}$ the residual conductivity from the unpaired quasiparticles follows the same non-Fermi liquid fractional power law, $\sigma_{1}(\omega) \propto \omega^{-1.2}$. (b), The plot for optimally-doped $\mathrm{YBa}_{2} \mathrm{Cu}_{3} \mathrm{O}_{6.95}\left(T_{c}=91 \mathrm{~K}\right)$, for light polarized along the $a$ axis, illustrating the fractional power law below $T_{c}$. (c), The plot for underdoped $\mathrm{YBa}_{2} \mathrm{Cu}_{3} \mathrm{O}_{6.60}\left(T_{c} \simeq 59 \mathrm{~K}\right)$, for light polarized along the $a$ axis, illustrating an identical (non-Fermi liquid) fractional power-law behavior in the normal (pseudogap) and superconducting states. (d), The plot for the $\mathrm{BaFe}_{2} \mathrm{As}_{2}\left(T_{\mathrm{SDW}}=138 \mathrm{~K}\right)$, for light polarized in the $a-b$ planes. Below $T_{S D W}$ the fractional power law $\sigma_{1} \propto \omega^{-1.2}$ is again observed.

formation of a pseudogap in the normal state ${ }^{19}$ and the commensurate reduction of the Fermi surface around the nodal regions, a condition that has been referred to as a 'nodal metal' ${ }^{20,21}$. The optical conductivity for this material is shown in Fig. 1c for light polarized along the $a$ axis. Just above $T_{c}$ in the normal state the low-frequency optical conductivity may be described using a non-Fermi liquid power-law, $\sigma_{1} \propto \omega^{-0.85}$; however, what is fascinating is that well below $T_{c}$ the response of the unpaired quasiparticles displays the identical fractional power law. This indicates the (unpaired) quasiparticles appear to behave the same way regardless of whether it is the pseudogap that results in the reduction of a large Fermi surface to a small arc or pocket $^{22}$, or the formation of a $d$-wave superconducting energy gap resulting in nodes. This non-Fermi liquid power-law behavior in the underdoped material has been previously observed in the microwave region $^{23}$; however, in that work the exponent is considerably larger, $\sigma_{1}$ $\propto \omega^{-1.45}$. The most likely source for this disagreement is the fact that the microwave experiments are done in the $\omega \tau \sim 1$ region, while the optical work was performed in the $\omega \tau \gg 1$ limit, suggesting that the relaxation processes in these two regimes may be different. Surprisingly, recent results on the single-layer, underdoped cuprate $\mathrm{HgBa}_{2} \mathrm{CuO}_{4+\delta}$ demonstrate that it displays Fermi liquid-like behavior $^{24}$, indicating that the nature of the underdoped (pseudogap) region in the cuprate materials is still controversial.

Interestingly, an almost identical behavior has also been observed in the $A \mathrm{Fe}_{2} \mathrm{As}_{2}(A=\mathrm{Ba}$ and $\mathrm{Ca})$ iron-arsenic compounds ${ }^{25}$. In $\mathrm{BaFe}_{2} \mathrm{As}_{2}$ a spin-density-wave (SDW) state develops below $T_{\text {SDW }} \simeq 138 \mathrm{~K}$, resulting in the formation of a Dirac-like cone in the electronic dispersion close to the Fermi surface ${ }^{26,27}$ with small pockets or puddles. The frequency-dependent scattering rate has a clear quadratic component just above $T_{\mathrm{SDW}}$, suggesting the nonmagnetic state of this material may be described as a Fermi liquid (see Supplementary Fig. S2a online); when the SDW transition is removed by Co substitution, the quadratic behavior persists from $295 \mathrm{~K}$ down to $27 \mathrm{~K}$ (see Supplementary Fig. S2b online). The optical conductivity of $\mathrm{BaFe}_{2} \mathrm{As}_{2}$ is shown in Fig. $1 \mathrm{~d}$; for $T \ll T_{\mathrm{SDW}}$ we once again observe the fractional power law in the residual low-frequency optical conductivity ${ }^{28}, \sigma_{1} \propto \omega^{-1.2}$, similar to that seen in the ground state of several of the cuprates. The identical power law is also observed in $\mathrm{CaFe}_{2} \mathrm{As}_{2}$ for $T \ll T_{\mathrm{SDW}}$ (see Supplementary Fig. S3 online).

\section{Discussion}

One important aspect of the fractional power law lies in its ability to remove the nodal quasiparticle (residual) response that masks the gap. In a superconductor, the real part of the optical conductivity at low frequencies may be expressed as the linear combination $\sigma_{1}(\omega)=\delta(0)+\sigma_{\mathrm{qp}}+\sigma_{\text {gap }}+\cdots$, where $\delta(0)$ is the zero-frequency component that corresponds to the superfluid density, $\sigma_{\mathrm{qp}}$ is the conductivity due to the unpaired quasiparticles, and $\sigma_{\text {gap }}$ is the contribution due to direct excitations across the gap. [In the normal state, $\delta(0)$ is absent and $\sigma_{\mathrm{qp}}$ is just the quasiparticle response from the whole Fermi surface.] Because we now have an explicit functional form for $\sigma_{\mathrm{qp}}$ for various materials, then for $\omega>0$ we neglect $\delta(0)$ and the low-frequency response is $\sigma_{\text {gap }} \simeq \sigma_{1}(\omega)-\sigma_{\mathrm{qp}}$. The conductivity due to the superconducting energy gap may be described phenomenologically using a Kubo-Greenwood approach ${ }^{29}$ in which all the zero-momentum transitions across the gap in the Brillouin zone are considered; in general terms, the optical conductivity due to the gap is a reflection of the joint density of states of the photo-excited electron and hole pairs. In a conventional superconductor with an isotropic energy gap $\Delta$ and weak coupling to phonons (or any other exchange boson), then for $T \ll T_{c}$ in systems at or close to the dirty limit $\left[1 / \tau_{0} \gtrsim 2 \Delta\right.$ where $\left.1 / \tau_{0}=1 / \tau(\omega \rightarrow 0)\right]$, the onset of absorption will occur at $2 \Delta$; for modest coupling, this onset shifts to $\Omega_{0}+2 \Delta$, where $\Omega_{0}$ is the energy of the boson ${ }^{30,31}$. Similarly, in a $d$-wave superconductor in the dirty limit with weak coupling, the onset would be expected at $\omega \simeq 0$; however, for moderate coupling the onset should shift to $\Omega_{0}$ with a local maximum at $\simeq \Omega_{0}+2 \Delta_{0}$.

The result for the removal of the quasiparticle contribution, $\sigma_{1}(\omega)$ $-\sigma_{\mathrm{qp}}$, is shown in Fig. 2 for $\mathrm{Bi}_{2} \mathrm{Sr}_{2} \mathrm{CaCu}_{2} \mathrm{O}_{8+\delta}$ at $\sim 6 \mathrm{~K}$, well below $T_{c}$ the resulting conductivity is effectively zero at low frequency and the onset of conductivity does not begin until $\omega \gtrsim 400 \mathrm{~cm}^{-1}$. This corresponds to the bosonic excitation at $\Omega_{0}$, the frequency above which a change occurs in the optical conductivity due to the strong renormalization of the scattering rate (see Fig. 1a). This indicates that there is at least moderate electron-boson coupling in this material ${ }^{32,33}$ and 


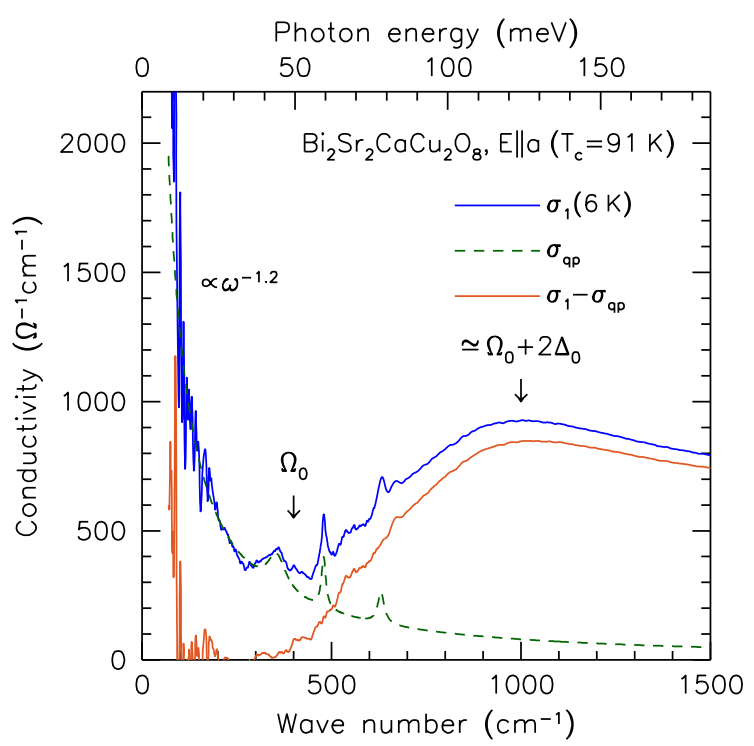

Figure $2 \mid$ The decomposition of the optical conductivity in a cuprate superconductor. The optical conductivity of optimally-doped $\mathrm{Bi}_{2} \mathrm{Sr}_{2} \mathrm{CaCu}_{2} \mathrm{O}_{8+\delta}$ at $6 \mathrm{~K}$ versus wave number (photon energy) with the residual quasiparticle conductivity shown and removed; several sharp features in the conductivity have been fit to Lorentzian oscillators (Supplementary Information) and have also been removed. The subtracted spectra shows an onset of absorption at $\Omega_{0}$ and a local maximum at $\simeq \Omega_{0}+2 \Delta_{0}$.

that the local maximum in the conductivity will be at $\simeq \Omega_{0}+2 \Delta_{0}$. The inferred values of $\Omega_{0} \simeq 50 \mathrm{meV}$ and $\Delta_{0} \simeq 35 \mathrm{meV}$ are in good agreement with estimates for these quantities based from angleresolved photoemission spectroscopy ${ }^{13}$, and are consistent with optical inversion techniques ${ }^{9,34}$. This procedure may also be successfully applied to the $\mathrm{YBa}_{2} \mathrm{Cu}_{3} \mathrm{O}_{6+y}$ materials (see Supplementary Fig. $\mathrm{S} 4$ online), as well as the iron-based $\mathrm{BaFe}_{2} \mathrm{As}_{2}$ and $\mathrm{CaFe}_{2} \mathrm{As}_{2}$ materials in their SDW states (see Supplementary Fig. S5 online).

The significance of our finding is the common fractional power law behavior of the low-frequency optical conductivity ( $\mathrm{THz}$ and farinfrared regions) in materials with Dirac cone-like electronic dispersion and nodal Fermi surfaces. More generally, the fractional power law behavior signals the importance of many-body effects in quantum materials with this unique electronic dispersion, where the fractional power law in conductivity is roughly equivalent to a nearly-linear frequency dependence of the scattering rate. Similar results have been found in single layer graphene in the linear dependence of the resistivity which is the result of electron-phonon (acoustic phonon) coupling ${ }^{35}$. However, in the materials discussed here, the electron-phonon coupling is weak. The power-law behavior observed in this work is likely the result of the scattering of nodal quasiparticles by low-energy (bosonic) excitations, or possibly some unique self-energy effect of the Dirac-like quasiparticles. What is common for these systems are the existence of antiferromagnetic spin fluctuations (or over-damped spin density waves in the SDW materials), which may be the underlying mechanism that gives rise to the nearly linear frequency dependence of the scattering rate.

\section{Methods}

The temperature dependence of the absolute reflectance was measured at a nearnormal angle of incidence over a wide frequency range using an in situ evaporation $\operatorname{method}^{36}$. In this study mirror-like as-grown crystal faces have been examined. The complex optical properties were determined from a Kramers-Kronig analysis of the reflectance ${ }^{37}$. The Kramers-Kronig transform requires that the reflectance be determined for all frequencies, thus extrapolations must be supplied in the $\omega \rightarrow 0, \infty$ limits. In the metallic state the low frequency extrapolation follows the Hagen-Rubens form, $R(\omega) \propto 1-\sqrt{\omega}$, while in the superconducting state $R(\omega) \propto 1-\omega^{4}$ is typically employed; however, it should be noted that when the reflectance is close to unity the analysis is not sensitive upon the choice of low-frequency extrapolation. The reflectance is assumed to be constant above the highest measured frequency point up to $\simeq 1 \times 10^{5} \mathrm{~cm}^{-1}$, above which a free electron gas asymptotic reflectance extrapolation $R(\omega) \propto 1 / \omega^{4}$ is employed ${ }^{38}$.

1. Gurzhi, R. N. Some features of the electrical conductivity of metals at low temperatures. Soviet Physics JETP 20, 953-960 (1965).

2. Nagel, U. et al. Optical spectroscopy shows that the normal state of $\mathrm{URu}_{2} \mathrm{Si}_{2}$ is an anomalous Fermi liquid. PNAS 109, 19161-19165 (2012).

3. van der Marel, D. Anisotropy of the optical conductivity of high- $T_{c}$ cuprates. Phys. Rev. B 60, R765-R768 (1999).

4. Dodge, J. S. et al. Low-frequency crossover of the fractional power-law conductivity in $\mathrm{SrRuO}_{3}$. Phys. Rev. Lett. 85, 4932-4935 (2000).

5. Orenstein, J. \& Millis, A. J. Advances in the physics of high-temperature superconductivity. Science 288, 468-474 (2000).

6. Dordevic, S. V. \& Basov, D. N. Electrodynamics of correlated electron matter. Ann. Phys. 15, 545-570 (2006).

7. Berthod, C. et al. Non-Drude universal scaling laws for the optical response of local Fermi liquids. Phys. Rev. B 87, 115109 (2013).

8. Basov, D. N. \& Timusk, T. Electrodynamics of high- $T_{c}$ superconductors. Rev. Mod. Phys. 77, 721-779 (2005).

9. Tu, J. J., Homes, C. C., Gu, G. D., Basov, D. N. \& Strongin, M. Optical studies of charge dynamics in optimally doped $\mathrm{Bi}_{2} \mathrm{Sr}_{2} \mathrm{CaCu}_{2} \mathrm{O}_{8+\delta}$. Phys. Rev. B 66, 144514 (2002).

10. Broun, D. M. What lies beneath the dome? Nat. Phys. 4, 170-172.

11. Buckley, R. G., Barowski, H. S. \& Renk, K. F. Far-infrared measurement of the dopant-dependent residual absorptivity of $\mathrm{Bi}_{2} \mathrm{Sr}_{2} \mathrm{CaCu}_{2} \mathrm{O}_{8+\delta}$. Phys. Rev. B 64, 014509 (2001).

12. Van Harlingen, D. J. Phase-sensitive tests of the symmetry of the pairing state in the high-temperature superconductors-Evidence for $d_{x^{2}-y^{2}}$ symmetry. Rev. Mod. Phys. 67, 515-535 (1995).

13. Damascelli, A., Hussain, Z. \& Shen, Z.-X. Angle-resolved photoemission studies of the cuprate superconductors. Rev. Mod. Phys. 75, 473-541 (2003).

14. Lee, P. A. Localized states in a $d$-wave superconductor. Phys. Rev. Lett. 71, 1887-1890 (1993).

15. Geim, A. K. \& Novoselov, K. S. The rise of graphene. Nat. Mater. 6, 183-191 (2007).

16. Moore, J. E. The birth of topological insulators. Nature 464, 194-198 (2010).

17. Shibauchi, T. et al. In-plane microwave conductivity and quasiparticle scattering rate of superconducting high- $\mathrm{T}_{c}$ cuprates. J. Phys. Soc. Jpn. 65, 3266-3273 (1996).

18. Homes, C. C. et al. Effect of $\mathrm{Ni}$ impurities on the optical properties of $\mathrm{YBa}_{2} \mathrm{Cu}_{3} \mathrm{O}_{6+y}$. Phys. Rev. B 60, 9782-9792 (1999).

19. Timusk, T. \& Statt, B. The pseudogap in high-temperature superconductors: an experimental survey. Rep. Prog. Phys. 62, 61-122 (1999).

20. Ando, Y., Lavrov, A. N., Komiya, S., Segawa, K. \& Sun, X. F. Mobility of the doped holes and the antiferromagnetic correlations in underdoped High- $T_{c}$ cuprates. Phys. Rev. Lett. 87, 017001 (2001).

21. Lee, Y. S. et al. Electrodynamics of the nodal metal state in weakly doped high- $T_{c}$ cuprates. Phys. Rev. B 72, 054529 (2005).

22. Kanigel, A. et al. Evolution of the pseudogap from Fermi arcs to the nodal liquid. Nature Phys. 2, 447-451 (2006).

23. Turner, P. J. et al. Observation of weak-limit quasiparticle scattering via broadband microwave spectroscopy of a $d$-wave superconductor. Phys. Rev. Lett. 90, 237005 (2003).

24. Mirzaei, S. I. et al. Spectroscopic evidence for Fermi liquid-like energy and temperature dependence of the relaxation rate in the pseudogap phase of the cuprates. PNAS 110, 5774-5778 (2013).

25. Johnston, D. C. The puzzle of high temperature superconductivity in layered iron pnictides and chalcogenides. Adv. Phys. 59, 803-1061 (2010).

26. Zabolotnyy, V. B. et al. $(\pi, \pi)$ electronic order in iron arsenide superconductors. Nature 457, 569-572 (2009).

27. Richard, P. et al. Observation of dirac cone electronic dispersion in $\mathrm{BaFe}_{2} \mathrm{As}_{2}$ Phys. Rev. Lett. 104, 137001 (2010).

28. Hu, W. Z. et al. Origin of the spin density wave instability in $A \mathrm{Fe}_{2} \mathrm{As}_{2}(A=\mathrm{Ba}, \mathrm{Sr})$ as revealed by optical spectroscopy. Phys. Rev. Lett. 101, 257005 (2008).

29. Harrison, W. A. Solid State Theory (McGraw-Hill, New York, 1970).

30. Akis, R., Carbotte, J. P. \& Timusk, T. Superconducting optical conductivity for arbitrary temperature and mean free path. Phys. Rev. B 43, 12804-12808 (1991).

31. Nicol, E. J., Carbotte, J. P. \& Timusk, T. Optical conductivity in high- $T_{c}$ superconductors. Phys. Rev. B 43, 473-479 (1991).

32. Carbotte, J. P., Schachinger, E. \& Basov, D. N. Coupling strength of charge carriers to spin fluctuations in high-temperature superconductors. Nature 401, 354-356 (1999).

33. Munzar, D., Bernhard, C. \& Cardona, M. Does the peak in the magnetic susceptibility determine the in-plane infrared conductivity of YBCO? A theoretical study. Physica C 312, 121-135 (1999).

34. Dordevic, S. V. et al. Extracting the electron-boson spectral function $\alpha^{2} F(\omega)$ from infrared and photoemission data using inverse theory. Phys. Rev. B 71, 104529 (2005).

35. Bolotin, K. I., Sikes, K. J., Hone, J., Stormer, H. L. \& Kim, P. Temperaturedependent transport in suspended graphene. Phys. Rev. Lett. 101, 096802 (2008). 
36. Homes, C. C., Reedyk, M., Crandles, D. A. \& Timusk, T. Technique for measuring the reflectance of irregular, submillimeter-sized samples. Appl. Opt. 32, 2976-2983 (1993).

37. Dressel, M. \& Grüner, G. Electrodynamics of Solids (Cambridge University Press, Cambridge, 2001)

38. Wooten, F. Optical Properties of Solids (Academic Press, New York, 1972).

\section{Acknowledgments}

The authors would like to acknowledge useful discussions with P.W. Anderson, Y.M. Dai, D.N. Basov, D.A. Bonn, S.V. Borisenko, G. Kotliar, P. Phillips, J.D. Rameau, D. Schmeltzer and C. Varma. Research supported by the U.S. Department of Energy, Office of Basic Energy Sciences, Division of Materials Sciences and Engineering under Contract No. DE-AC02-98CH10886. C.C.H. would like to acknowledge the hospitality of the Theory Institute for Strongly Correlated and Complex Systems.

\section{Author contributions}

C.C.H. supervised the project and wrote the manuscript, and performed the optical measurements on hole-doped $\mathrm{YBa}_{2} \mathrm{Cu}_{3} \mathrm{O}_{6+y}$ and electron-doped $\mathrm{Pr}_{1.85} \mathrm{Ce}_{0.15} \mathrm{CuO}_{4}$. J.J.T. supervised part of the project, measured the optical properties of $\mathrm{Bi}_{2} \mathrm{Sr}_{2} \mathrm{CaCu}_{2} \mathrm{O}_{8+\delta}$ and $\mathrm{BaFe}_{1.85} \mathrm{Co}_{0.15} \mathrm{As}_{2}$, and wrote part of the manuscript. G.D.G. synthesized single crystals of $\mathrm{Bi}_{2} \mathrm{Sr}_{2} \mathrm{CaCu}_{2} \mathrm{O}_{8+\delta}$. A.A. measured the optical properties of $\mathrm{BaFe}_{2} \mathrm{As}_{2}$ and $\mathrm{CaFe}_{2} \mathrm{As}_{2} ;$ J.L. assisted with the preparation of the manuscript.

\section{Additional information}

Supplementary information accompanies this paper at http://www.nature.com/ scientificreports

Competing financial interests: The authors declare no competing financial interests.

How to cite this article: Homes, C.C., Tu, J.J., Li, J., Gu, G.D. \& Akrap, A. Optical conductivity of nodal metals. Sci. Rep. 3, 3446; DOI:10.1038/srep03446 (2013).

(c) (i) (-) $\odot$ This work is licensed under a Creative Commons Attribution-

cc. visit http://creativecommons.org/licenses/by-nc-nd/3.0 